\title{
SINGULAR CONVOLUTION OPERATORS ON THE HEISENBERG GROUP ${ }^{1}$
}

\author{
BY D. GELLER AND E. M. STEIN
}

1. Statement of results and outline of method. The purpose of this note is to announce results dealing with convolution operators on the Heisenberg group. As opposed to the well-known situation where the kernels are homogeneous and $C^{\infty}$ away from the origin, the kernels we study are homogeneous but have singularities on a hyperplane. Convolution operators with such kernels arise in the study of the $\bar{\partial}$-Neumann problem, as we indicate below. The main feature of our study will be an analysis which has no direct analogue in the case of the usual (abelian) convolution operators, but is instead based on the noncommutative Fourier analysis of the Heisenberg group.

Let $\mathbf{H}^{n}$ denote the Heisenberg group, the Lie group with underlying manifold $\mathbf{C}^{n} \times \mathbf{R}$ and multiplication $(z, t) \cdot\left(z^{\prime}, t^{\prime}\right)=\left(z+z^{\prime}, t+t^{\prime}+2 \operatorname{Im} z \cdot \bar{z}^{\prime}\right)$, where $z \cdot \bar{z}^{\prime}=\Sigma z_{j} \bar{z}_{j}^{\prime}$. $\mathbf{H}^{n}$ possesses dilations: let $D_{r}(z, t)=\left(r^{2} z, r t\right)$. A function $f$ is homogeneous of degree $k$ if $f \circ D_{r}=r^{k} f$, and there is a dual notion for distributions. Suppose $K^{\prime}$ is a homogeneous distribution of degree $-2 n-2$ which agrees with a function away from the origin. Assume that this function is smooth on $\mathbf{H}^{n}-\{0\}$, or more generally that it satisfies an $L^{1}$-Dini condition there. Then it is known [3, Theorem 2.1] that the convolution operator $A^{\prime}$ : $C_{0}^{\infty}\left(\mathbf{H}^{n}\right)-\rightarrow C^{\infty}\left(\mathbf{H}^{n}\right)$ given by $A^{\prime} f=f * K^{\prime}$ extends to a bounded operator from $L^{p}$ to $L^{p}$ for $1<p<\infty$ if it is bounded on $L^{2}$. Here $*$ denotes group convolution.

It is our intention to study more singular convolution operators. Thus, let $J(z)$ be a homogeneous distribution of degree $-2 n$ on $\mathbf{C}^{n}$, which agrees with a smooth function away from the origin, and define the distribution $K$ on $\mathbf{H}^{n}$ by $K(z, t)=J(z) \delta(t)$. Here $\delta(t)$ is the Dirac delta function in the $t$ variable. Then $K$ is homogeneous of degree $-2 n-2$ and we assert

Theorem. The operator $A: C_{0}^{\infty}\left(\mathbf{H}^{n}\right) \rightarrow C^{\infty}\left(\mathbf{H}^{n}\right)$ given by $A f=f * K$ extends to a bounded operator from $L^{p}$ to $L^{p}$ for $1<p<\infty$.

The analogue of the theorem for Euclidean convolution is immediate, since one can convolve on each hyperplane $t=$ constant separately. No such argument is available for $\mathbf{H}^{n}$. Our strategy in proving the theorem is to use

Received by the editors August 11, 1981.

1980 Mathematics Subject Classification. Primary 42B20, 43A30, 35N15.

1 Research supported by the National Science Foundation.

(c) 1982 American Mathematical Society 0002-9904/82/0000-0145/\$02.25 
interpolation, and as such we shall study convolution operators on $L^{2}$ which are even more singular than $A$, operators whose Euclidean analogues are unbounded on $L^{2}$. As the simplest example of this, we make a key observation.

ObSERVATION. The operator $B: C_{0}^{\infty}\left(\mathbf{H}^{n}\right) \rightarrow C^{\infty}\left(\mathbf{H}^{n}\right)$ given by $B f=f * T^{n} \delta(t)$ extends to a bounded operator on $L^{2}$; in fact it is unitary up to a constant multiple. Here $T=\partial / \partial t$.

Proof. For $f \in C_{0}^{\infty}, T^{n} f * \delta(t)=\int_{\mathrm{C}^{n}} T^{n} f\left(z-z^{\prime}, t-2 \operatorname{Im} z \cdot \bar{z}^{\prime}\right) d z^{\prime} d \bar{z}^{\prime}$ $=\int_{\mathrm{C}^{n}} T^{n} f\left(z^{\prime}, t+2 \operatorname{Im} z \cdot \bar{z}^{\prime}\right) d z^{\prime} d \bar{z}^{\prime}$. Let $\bar{F}_{c}$ denote Fourier transform in $t$ alone, and $F$ Euclidean Fourier transform in both $t$ and $z$. We have

$$
\begin{aligned}
F_{c}(B f)(z, \lambda) & =\int_{-\infty}^{\infty} e^{i \lambda t} B f(z, t) d t=(-i \lambda)^{n} \int_{\mathrm{C}^{n}} e^{-4 i \lambda \operatorname{Im} z \cdot \bar{z}^{\prime}} F_{c} f\left(z^{\prime}, \lambda\right) d z^{\prime} d \bar{z}^{\prime} \\
& =(-i \lambda)^{n} F f(2 i \lambda z, \lambda)
\end{aligned}
$$

since $2 i \lambda \operatorname{Im} z \cdot \bar{z}^{\prime}=\operatorname{Re}(2 i \lambda z) \cdot \bar{z}^{\prime}$. Then $\|B f\|_{2}^{2}=(2 \pi)^{-1}\left\|F_{c}(B f)\right\|_{2}^{2}=$ $(2 \pi)^{-(2 n+1)} 2^{-2 n}\|f\|_{2}^{2}$ as required.

In fact the same proof gives the same result with $T^{n} \delta(t)$ replaced by $T^{n} \delta(t-\psi(z))$ where $\psi: \mathbf{C}^{n} \rightarrow \mathbf{R}$ is any measurable function.

If one makes the reasonable assumption that a convolution operator with singularity weaker than $B$ is also bounded on $L^{2}$, one can hope to prove the theorem as follows. Consider the distribution $g_{\gamma}(t)=\Gamma(\gamma / 2)^{-1}|t|^{-1+\gamma}$ on $C_{0}^{\infty}(\mathbf{R})$. Here $\gamma \in \mathrm{C}, \operatorname{Re} \gamma>0$. Now $g_{\gamma}$ has an analytic continuation to all $\gamma \in \mathbf{C}$, and for $k \in \mathbf{Z}^{+}, g_{\gamma} \rightarrow c_{k} T^{2 k} \delta(t)$ as $\gamma \rightarrow-2 k$, where $c_{k} \neq 0$. In the notation of Theorem 1, suppose $J(z)=$ P.V. $\left(\Omega(z) /|z|^{2 n}\right)$ and let us set $K_{\gamma}(z, t)=$ P.V. $\left[\Omega(z) g_{\gamma}(t) /\left(|z|^{4}+t^{2}\right)^{(n+\gamma) / 2}\right]$. Further let $A_{\gamma}$ denote the operation of convolution with $K_{\gamma}$. Then $K_{0}=K$. Motivated by the above considerations we expect that $A_{\gamma}$ is bounded on $L^{2}$ for $-n \leqslant \operatorname{Re} \gamma$. If $\operatorname{Re} \gamma>0$, the $L^{1}$-Dini condition is satisfied and therefore $A_{\gamma}$ is bounded on $L^{p}$. Interpolation then would prove the theorem. (It would also give the by-product that, if $-n<\operatorname{Re} \gamma<0$, $A_{\gamma}$ is bounded on a certain range of $L^{p}$-spaces.)

2. The group Fourier transform. To carry out this plan to prove the theorem, it will suffice to examine the group Fourier transform (F.T.) of a certain modification of $K_{\gamma}$, for $-n \leqslant \operatorname{Re} \gamma$. We discuss this F.T.

For each $\lambda \in \mathbf{R}^{*}(=\mathbf{R}-\{0\})$ let $H_{\lambda}$ be a separable Hilbert space with fixed orthonormal basis $\left\{E_{\alpha, \lambda}\right\}_{\alpha \in\left(\mathbf{Z}^{+}\right)^{n}}$ where $\mathbf{Z}^{+}=\{0,1,2, \ldots\}$. On the algebraic span of this basis, we define the weighted shift (annihilation and creation) operators $W_{k \lambda}, W_{k \lambda}^{+}$for $1 \leqslant k \leqslant n$ as follows:

$$
\begin{aligned}
W_{k} E_{\alpha} & =\left(2 \alpha_{k}|\lambda|\right)^{1 / 2} E_{\alpha-e_{k}}, \quad \text { zero if } \alpha_{k}=0 . \\
W_{k}^{+} E & =\left[2\left(\alpha_{k}+1\right)|\lambda|\right]^{1 / 2} E_{\alpha+e_{k}},
\end{aligned}
$$


for $\lambda>0$. The right sides are to be reversed if $\lambda<0$. Here and elsewhere we frequently drop the $\lambda$ subscript.

One may extend $W_{k}, W_{k}^{+}$to a domain on which $-z \cdot W^{+}+\bar{z} \cdot W$ is essentially skew-adjoint for all $z \in \mathbf{C}^{n}$, so that $V_{z}^{\lambda}=\exp \left[-z \cdot W^{+}+\bar{z} \cdot W\right]$ is unitary on $H$. The Weyl transform $G_{\lambda}: L^{1}\left(\mathbf{C}^{n}\right) \rightarrow B\left(H_{\lambda}\right)$ is defined by $G_{\lambda} F=$ $\int_{\mathrm{C}^{n}} V_{z}^{\lambda} F(z) d z d \bar{z}$.

One can prove [2] an analogue of Hecke's identity (see e.g. [5]) for G, as follows. Let $P=\Sigma_{|\rho|=p,|\gamma|=q} a_{\rho \gamma} z^{\rho} \bar{z}^{\gamma}$ be a harmonic polynomial on $\mathbf{C}^{n}$ of bidegree $(p, q)$. Set $\kappa=p+q$, and

$$
W P=\sum a_{\rho \gamma} W^{\rho}\left(W^{+}\right)^{\gamma}=\sum a_{\rho \gamma}\left(W^{+}\right)^{\gamma} W^{\rho} .
$$

For $a>0$, put $F(z)=e^{-a|z|^{2}} P(z)$. Then

$$
(G F) E_{\alpha}=(-1)^{q} \pi^{n}[(1+\sigma)|\lambda|]^{-(n+\kappa)}(W P) s^{N-p^{\prime}} E_{\alpha} .
$$

Here $\sigma=a /|\lambda| ; s=(\sigma-1) /(\sigma+1) ; p^{\prime}=p$ if $\lambda>0, q$ if $\lambda<0$, and $N=|\alpha|$. Note that $(G F) E_{\alpha}=0$ if $N<p^{\prime}$, for then $W(P)$ shifts $E_{\alpha}$ to zero.

The F.T. on $\mathbf{H}^{n}$ is defined as follows. For $\lambda \in \mathbf{R}^{*}$, define $F_{c}^{\lambda}: L^{1}\left(\mathbf{H}^{n}\right)$ $\rightarrow L^{1}\left(\mathbf{C}^{n}\right)$ by $\left(F_{c}^{\lambda} f\right)(z)=\int_{--\infty}^{\infty} e^{i \lambda t} f(z, t) d t$. Then $\hat{f}$, the F.T. of $f$, is defined to be the family of operators $(\hat{f}(\lambda))$ where $\hat{f}(\lambda)=G_{\lambda}\left(F_{c}^{\lambda} f\right)$.

To prove the theorem we use a consequence of (2) to demonstrate the boundedness of the F.T. of a modification of the $K_{\gamma}$ given above. To see the relevance of (2), note for example,

$$
\left(T^{n} \delta(t) \hat{)}(\lambda) E_{\alpha}=(-i \lambda)^{n} G_{\lambda}(1) E_{\alpha}=(-\pi i \operatorname{sgn} \lambda)^{n}(-1)^{N} E_{\alpha}\right.
$$

by (2), giving another proof of the Observation. For another consequence of (2), write $w=|z|^{2}-i t, \bar{w}=|z|^{2}+i t$. Then [2]: if $P$ is as in (2), $-1<s<1$, $f(z, t)=(s w+\bar{w})^{-k} P(z)$, then

(3) $\hat{f}(\lambda) E_{\alpha}=(-1)^{q} \pi^{n+1} 2^{1-n-k} \Gamma(n+\kappa-j+1)^{-1}|\lambda|^{-j}(1-s)^{j-1} \omega(P) s^{N-p^{\prime}} E_{\alpha}$

if $\lambda>0 ; \hat{f}(\lambda)=0$ if $\lambda<0$. The formulae for $\lambda>0, \lambda<0$ are reversed if we replace $s w+\bar{w}$ by $s \bar{w}+w$. Here $j+k=n+\kappa+1$ and $0<k \leqslant n+\kappa / 2+1$ with $f$ interpreted as a PV distribution when $k=n+\kappa / 2+1$. Note that (3) is particularly useful when one can write

$$
\begin{aligned}
K^{\prime} & =\left[\int_{0}^{\infty}(s w+\bar{w})^{-k} f(s) d s\right] P(z) \\
& =\left[\int_{0}^{1}(s w+\bar{w})^{-k} f(s)+\int_{0}^{1}(s \bar{w}+w)^{-k} g(s) d s\right] P(z)
\end{aligned}
$$


where $g(s)=f(1 / s) s^{k-2}$. Note that the right side of (4) is not directly defined for $z=0$, for then $s w+\bar{w}=0$ when $s=1$. Generally speaking, the behavior of $K$ as $z \rightarrow 0$ is the same as that of $f$ as $s \rightarrow 1$. Thus $K^{\prime}$ is (resp.) smooth or real analytic if and only if $f$ is (resp.) smooth or real analytic at 1. As an example of an application of formula (4) and its F.T., the case $f(s)=s^{\gamma-1}, k=$ $n+\kappa / 2$ yields (see [2]) the fundamental solution to the differential operators $L_{\alpha}$ given in [1].

Returning now to the notation of $\S 1$, let us assume $\Omega(z)=P(z /|z|)-$ otherwise one must expand $\Omega$ as an infinite sum. We shall write our modification of $K_{\gamma}$ essentially in the form $\left[\int_{-\infty}^{0}(s w+\bar{w})^{-(n+1+\kappa / 2)} f(s) d s\right] P(z)$. Since $s w+\bar{w}=0$ in the integrand only if $t=0$ and $s=-1$, we expect to capture the behavior of $K_{\gamma}$ as $t \rightarrow 0$ in the behavior of $f$ at $s=-1$.

Briefly, then, we are able to prove the theorem by computing and estimating the F.T. of

(5) $\left[\int_{-1}^{0}(s w+\bar{w})^{-(n+1+\kappa / 2)} f_{\gamma}(s) d s+\int_{-1}^{0}(s \bar{w}+w)^{-(n+1+\kappa / 2)} f_{\gamma}(s) d s\right] P(z)$ where $f_{\gamma}(s)=(1+s)^{n+\gamma+\kappa / 2-1}(1-s)^{-\gamma}$, since as can be shown (5) behaves essentially like $K_{\gamma}$.

3. Applications. Let $J_{\epsilon}(z)=J(z)$, when $|z|>\epsilon$, and $J_{\epsilon}(z)=0$ otherwise, where $J$ is as in the theorem. Define $K_{\epsilon}(z, t)=J_{\epsilon}(z) \delta(t)$, and $A_{\epsilon}(f)=f * K_{\epsilon}$. A simple modification of the proof of the theorem then gives us the following:

COROLlary. The operators $A_{\epsilon}$ are bounded on $L^{p}, 1<p<\infty$, with norms independent of $\epsilon$.

Operators closely related to those treated here arise in the $\bar{\partial}$-Neumann problem. This reduction and its consequences are studied in [4], so we shall content ourselves here with one illustration. One wishes to prove that the operator

$$
f \rightarrow \int_{0}^{\infty} \int_{\mathrm{H}^{n}} L\left(y^{-1} \cdot x, \rho+\mu\right) f(y, \mu) d y d \mu
$$

maps $L^{p}\left(\mathbf{H}^{n} \times \mathbf{R}^{+}\right)$into itself, where e.g.

$$
L(x, \rho)=z_{j} z_{k}\left(|z|^{2}+t^{2}+\rho^{2}\right)^{-n-1}\left(|z|^{2}+\rho-i t\right)^{-2} .
$$

This is then reducible to the uniform boundedness (on $L^{p}\left(\mathbf{H}^{n}\right)$ ) of the operators of convolution with $z_{j} z_{k}\left(|z|^{2}+\epsilon^{2}\right)^{-n-1} \delta(t)$, which in turn is reducible to the corollary. 


\section{REFERENCES}

1. G. B. Folland and E. M. Stein, Estimates for the $\bar{\partial}_{b}$ complex and analysis on the Heisenberg group, Comm. Pure. Appl. Math. 27 (1974), 429-522.

2. D. Geller, Spherical harmonics, the Weyl transform and the Fourier transform on the Heisenberg group (to appear).

3. A. Korányi and S. Vagi, Singular integrals on homogeneous spaces and some problems of classical analysis, Ann. Scuola Norm. Sup. Pisa 3 (25) (1971), 575-648.

4. D. H. Phong and E. M. Stein (to appear).

5. E. M. Stein and G. Weiss, Introduction to Fourier analysis on Euclidean spaces, Princeton Univ. Press, Princeton, N.J., 1971.

DEPARTMENT OF MATHEMATICS, STATE UNIVERSITY OF NEW YORK AT STONY BROOK, STONY BROOK, NEW YORK 11794

DEPARTMENT OF MATHEMATICS, PRINCETON UNIVERSITY, PRINCETON, NEW JERSEY 08544 
